\title{
DARK MATTER SEARCH WITH DIRECTION SENSITIVE SCINTILLATORS
}

\author{
H. SEKIYA \\ Department of Physics, School of Science, Kyoto University \\ Kitashirakawa, Sakyo, Kyoto 606-8502, Japan \\ E-mail: sekiya@cr.scphys.kyoto-u.ac.jp \\ M. MINOWA, Y. SHIMIZU, W. SUGANUMA \\ Department of Physics, School of Science, University of Tokyo \\ 7-3-1 Hongo Bunkyo-ku, Tokyo, 113-0033 \\ Y. INOUE \\ International Center for Elementary Particle Physics, University of Tokyo \\ 7-3-1 Hongo Bunkyo-ku, Tokyo, 113-0033
}

\begin{abstract}
We have carried out the dark matter search with a $116 \mathrm{~g}$ direction-sensitive stilbene crystal in Kamioka Observatory. With the crystal fixed to the earth, we searched the modulation of the light output. No modulation signal was found due to the small size of the detector crystal and the higher background rate yet to be eliminated. However, it demonstrated the effectiveness of the method of direction sensitive search for the dark matter with an implementation of the anisotropic organic scintillation crystal.
\end{abstract}

\section{Introduction}

The most convincing signature of the WIMPs appears in the direction of nuclear recoils induced by WIMPs ${ }^{1}$. Although studies on detecting the signature by measuring the recoil directions have been carried out ever since it was indicated to be a reliable method, no dark matter search had been conducted with direction sensitive detector because of its difficulties. Recoil energy is only a few tens of $\mathrm{keV}$ and the track length of recoil nucleus should be short. Consequently, low pressure TPC have been studied as the directional WIMP detector principally, such as DRIFT and NEWAGE ${ }^{2}$. However, realistic experiments with gaseous detectors are very challenging because they are required the large fiducial volume and the great stability 

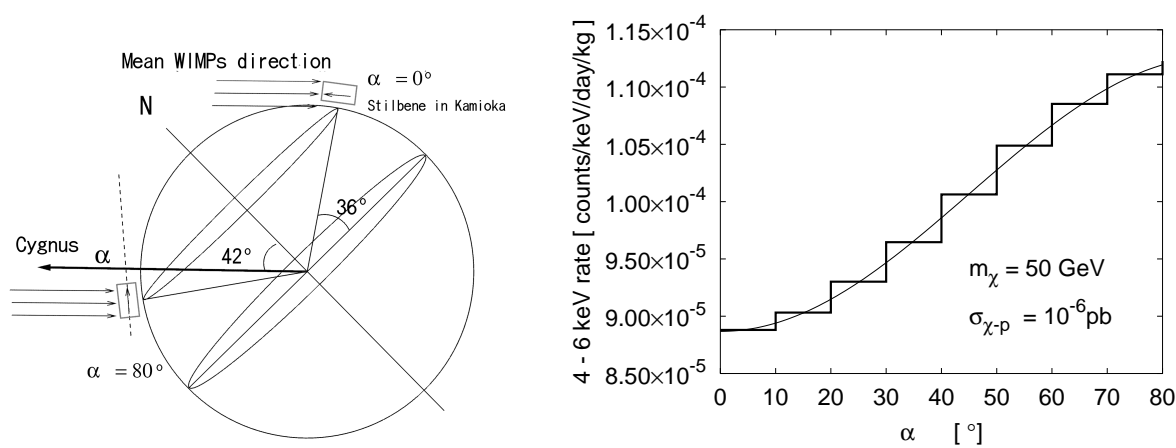

Figure 1. Left: Schematic drawing of the experimental approach mentioned in the text. Right: The expected event rate for $4-6 \mathrm{keV}$ as a function of $\alpha$. The thin line shows $A-S \cos 2 \alpha$, where $S=1.20 \times 10^{-5}$ counts $/ \mathrm{keV} /$ day $/ \mathrm{kg}$ and $A=1.01 \times 10^{-4}$ counts $/ \mathrm{keV} / \mathrm{day} / \mathrm{kg}$ in this case. The parameters that we used in the calculation are $\rho_{0}=0.3 \mathrm{GeV} / \mathrm{cm}^{3}, v_{0}=220 \mathrm{~km} / \mathrm{sec}$, WIMP-proton spin independent cross section $\sigma_{\chi-p}=10^{-6} \mathrm{pb}$, and $m_{\chi}=50 \mathrm{GeV}$.

with very fine resolution. Therefore, it is significant to explore alternative experimental approach.

It is known that scintillation efficiency of organic crystals to heavy particles depends on the direction of the particles with respect to the crystallographic axes. This property makes it possible to propose a WIMP detector sensitive to the recoil direction of the nucleus ${ }^{3}$.

We measured the carbon recoils in a stilbene crystal for recoil energies of $30 \mathrm{keV}$ to $1 \mathrm{MeV}$ and shown that the scintillation efficiency does vary by $7 \%$ depending on the direction of the recoil carbon with respect to $c^{\prime}$ axis ${ }^{4}$.

Then, we estimated the response to WIMPs when stilbene crystals installed in Kamioka ${ }^{5}$. As illustrated in Fig. 1, a suitable arrangement for the stilbene crystal is to fix the detector with the $c^{\prime}$ axis in parallel to the horizontal plane and towards the North assuming the WIMP halo is an isothermal sphere. In that case, the mean incident angle of the WIMP with respect to $c^{\prime}$ axis, $\alpha$, varies about $80^{\circ}$ within a sidereal daily period.

The expected event rate for 4-6 $\mathrm{keV}$ region as a function of $\alpha$ calculated by Monte Carlo method is shown in Fig. 1. As indicated, the variation can be well fitted by a function $A-S \cos 2 \alpha$.

As the next step, we have performed a pilot experiment at Kamioka to prove the feasibility of this method. In this paper, we report on the measurement and its results. 


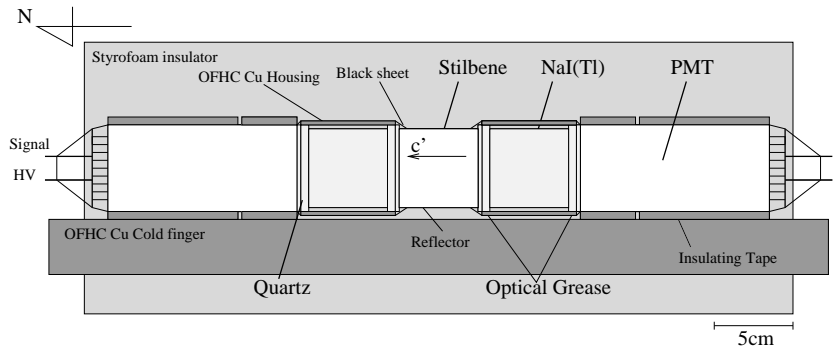

Figure 2. Schematic view of the detector setup.

\section{The Experimental Setup}

The schematic view of the detector assembly is shown in Fig. 2. The $\phi 50 \mathrm{~mm} \times 50 \mathrm{~mm}(116 \mathrm{~g})$ cylindrical stilbene crystal is viewed by two Hamamatsu R8778MOD low background PMTs through two Horiba low background $\mathrm{NaI}(\mathrm{Tl})$ active shields. Self coincidence of two PMTs are required and both PMTs are cooled down at about $-7^{\circ} \mathrm{C}$ to reduce dark current further.

The detector assembly is shielded with $10 \mathrm{~cm}$ OFHC copper, $15 \mathrm{~cm}$ Lead, and $20 \mathrm{~cm}$ polyethylene. The EVOH sheets are formed into air tight bags filled with nitrogen gas for purging the radon gas.

The whole setup is laid with the $c^{\prime}$ axis of the stilbene crystal parallel to the north-south direction.

\section{Measurement Results}

With the detector system, we started the measurement in October 25, 2003 and it was halted in December 11, 2003 . The obtained energy spectrum with the stilbene is shown in Fig. 3.

The background event rate is as high as 2000 counts/keV/day/kg for 4-6 keV region, however, the event rate of WIMPs should change in a cycle of one sidereal day -i.e. 23.934 hours - independent of halo models. Therefore, in order to search the modulation signal in frequency domain, we derived the power spectrum of the time data of the event rate for 4-6 $\mathrm{keV}$ region during the measurements ${ }^{5}$ using Lomb's periodgram method ${ }^{6}$. Fig. 4 shows the power spectrum for the frequency interval $0-0.3$ hour $^{-1}$.

The signal with the frequency of $1 / 23.934$ hours cannot be discerned from Fig. 4. That means the isotropic "white noise" events dominates the high rates of background events which should be eliminated. 


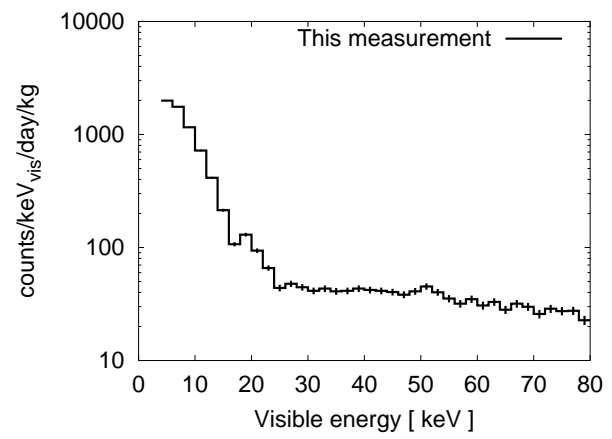

Figure 3. Low energy spectrum obtained with the stilbene crystal.

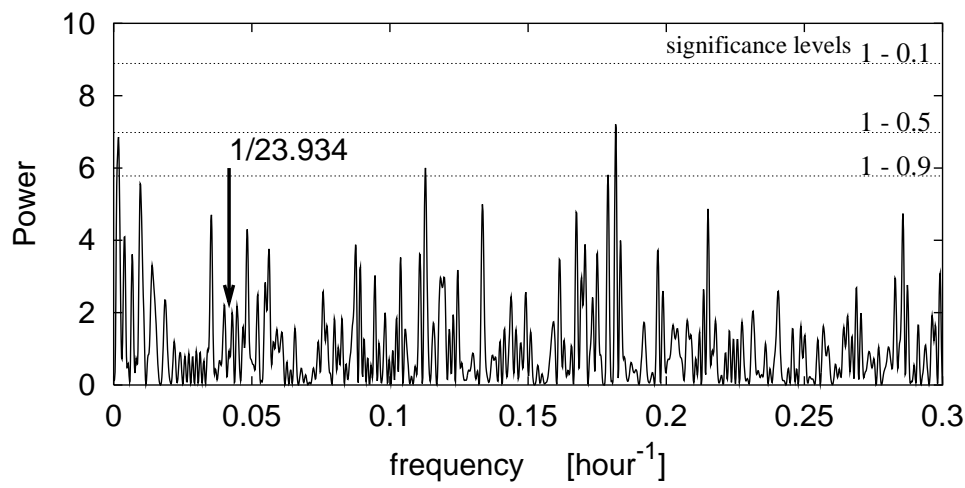

Figure 4. Power spectrum of the time data of event rate for $4-6 \mathrm{keV}$ in the stilbene.

For all that, we derived limits on cross sections from this isotropic results. Fig. 5 shows the measured event rate for $4-6 \mathrm{keV}$ as a function of $\alpha$. As indicated in Fig. 1, the event rate should vary as $A-S \cos 2 \alpha$, and both $A$ and $S$ are in proportion to $\sigma_{\chi-p}$. Accordingly, from the measured unmodulated part, $A$, conventional limits can be derived, and from the measured modulation amplitude, $S$, limits of direction sensitivity can be derived. The obtained limits on $\sigma_{\chi-p}$ are shown in Fig. 6. The limit is far looser than the contemporary non-directional limits, however, it is the limit from the directional signature of WIMPs. In addition, as the directional limit is derived from the "signal of WIMPs", the better limits will be obtained with the higher statistics. 


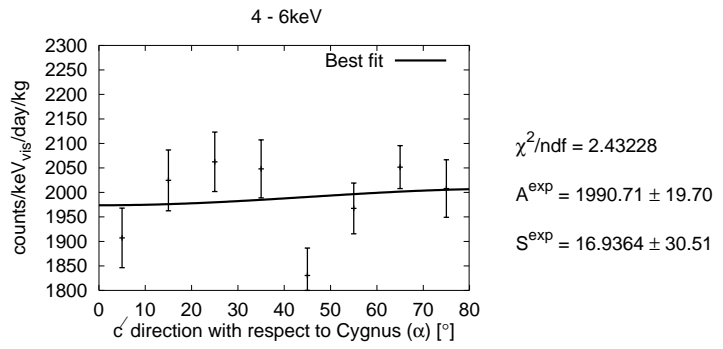

Figure 5. The measured event rate for $4-6 \mathrm{keV}$ as a function of $\alpha$. Expected $A-S \cos 2 \alpha$ is fitted.

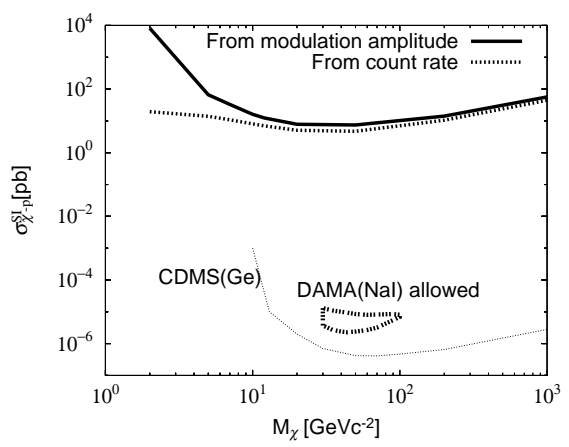

Figure 6. The obtained $\sigma_{\chi-p}$ limits as a function of WIMP mass $M_{\chi}$.

Fig. 7 indicates the background and exposure dependence of the achievable limits on $\sigma_{\chi-p}$. We see from Fig. 7 that the directional limit will be comparable to contemporary non-directional limit if we achieved the background level as low as $10^{-3}$ counts $/ \mathrm{keV} / \mathrm{day} / \mathrm{kg}$. Stilbene crystals could potentially detect the robuster WIMP signal arising from the earth's rotation in our galaxy as compared with the annual modulation signal.

\section{Discussions and Prospects}

It is obvious that rather high background rate due to the radioactivity in PMTs limits the sensitivity. The small light yield of the stilbene is another essential problem. Therefore, in order to overcome the difficulties, highly radio-pure, high quantum efficiency, high gain photon detector is indispensable. In that respect, we focused on Avalanche photodiodes(APD). 


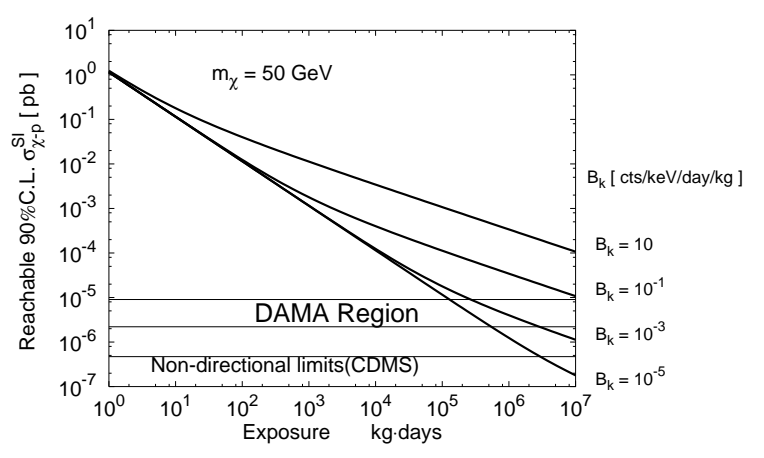

Figure 7. Background rate dependence of the expected sensitivity.

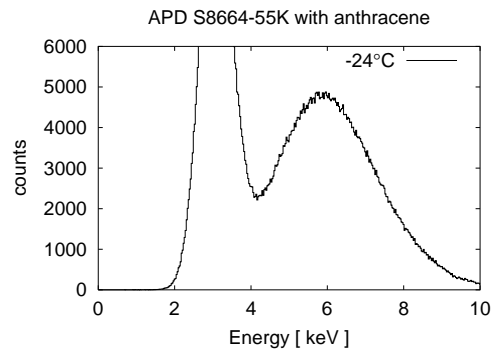

Figure 8. An example of the performance of the HAMAMATSU S8664-55K. $5.9 \mathrm{keV}$ X-ray spectrum measured at $-24^{\circ} \mathrm{C}$ with anthracene crystal $\left(15 \times 15 \times 15 \mathrm{~mm}^{3}\right)$.

Fig. 8 shows an example of the performance of an APD (HAMAMATSU S8664-55K) with anthracene crystal. $5.9 \mathrm{keV}$ X-rays from ${ }^{55} \mathrm{Fe}$ are clearly resolved. This result suggests that APDs will be promising devices for organic scintillators and will pull up the potential of the direction sensitivity.

\section{References}

1. D.N. Spergel, Phys. Rev. D 37, 1353 (1988).

2. N.J.C. Spooner - these proceedings; K. Miuchi - these proceedings.

3. Y. Shimizu et al., Nucl. Instr. and Meth. A 469, 347 (2003); R. Bernabei et al., Eur. Phys. J. C 28, 203 (2003); N.J.C. Spooner et al., International Workshop on Identification of Dark Matter, (World Scientific 1997), p481.

4. H. Sekiya et al., Phys. Lett B 571, 132 (2003).

5. H. Sekiya et al., astro-ph/0405598, To appear in the proceedings of the 5th Workshop on "Neutrino Oscillations and their Origin" (NOON2004)

6. Y. Ramachers, Astropart. Phys 19, 419 (2003). 